\title{
IMPROVED APIT Algorithm and Its Application in Core Poisoning Systems
}

\author{
Zhiyi Fang ${ }^{1, a}$, Hongyu Sun ${ }^{1, b}$, Zelin Deng ${ }^{2, ~ c}$, Fan Yang ${ }^{2, d}$ \\ ${ }^{1}$ College of Computer Science and Technology Jilin University Changchun, 130012, P.R. China \\ ${ }^{2}$ Beijing ACEWAY Telecom Technology Co., Ltd. \\ afangzy@jlu.edu.cn, ${ }^{\text {b }}$ shy12@mails.jlu.edu.cn, ${ }^{\mathrm{c}}$ dengzl@aceway.com.cn, ${ }^{\mathrm{d}} \mathrm{y}$ angfan@aceway.com.cn
}

Keywords: WSN, APIT, IMPROVED APIT

\begin{abstract}
Wireless sensor network (WSN) is widely used in intelligent medical, coal monitoring and etc., and poisoning is one of the most important technologies of WSN. APIT (approximate point in time) is one of the efficient poisoning algorithm in WSN, the advantage of APIT is the localization error rate is lower than other distance-free algorithms, so, we select APIT as the poisoning algorithm in core poisoning system, but according to the special application, there are some disadvantage of APIT algorithm, firstly, the cover rate can't reach $100 \%$; secondly, the core well is a C-shape like distribution instead of a regular rectangle distribution. According to the disadvantages of APIT in core poisoning systems, IMPROVED APIT is proposed in this paper, experimental research shows that IMPROVED APIT algorithm has a lower localization cover rate and a higher cover rate in C-shape distributions compared with APIT algorithm.
\end{abstract}

\section{Introduction}

WSN (Wireless sensor network) is a kind of self-organized network, the node of which is not expensive, and the size of the node is small, the energy of the node is limited [1]. But as the development of ToI (things of internet), wireless sensor network is used widely in all kinds of regions, such as environment monitoring, agriculture monitoring, military affairs, and intelligence medical and etc... But the poisoning algorithms play a crucial role in WSN, such as the routing algorithm which is related about geographic depends on the location of the each node [2]. Currently, the classic localization algorithm is divided into two kinds: distance-based and distance-free algorithm, and the distance-free algorithms is widely used in most of the applications such as core monitoring and poisoning systems because the cost of the node is lower and the efficiency of the them is higher. There are four kinds of distance-free algorithm; they are Centroid algorithm, DV-Hop algorithm, APIT algorithm, and Amorphous algorithm [3].

This paper focusing the specific application of core poisoning systems, the requirement of the core poisoning is low localization error rate and high cover rate. Experiments and other researchers conclusion shows that APIT algorithm has the lowest localization error rate compared with other localization algorithm [4]. So this paper choose APIT algorithm as the localization algorithm, but there still exists disadvantage of APIT when it used in core poisoning systems. The reason is that the distribution in core well is mainly as a C-shape instead of the usually Rectangle-like distribution, the cover rate and localization error rate of APIT in C-shape distribution is not that in Rectangle-like distributions.

In order to solve the problem above, this paper proposed an improved APIT algorithm called IMPROVED APIT. This algorithm aims to make the cover rate higher and localization error rate lower. So it can be more suitable for the application of core poisoning systems.

\section{APIT algorithm and its performance analysis}

APIT [5, 6] (Approximate Point in Test) algorithm is a distance-free WSN localization algorithm which is proposed by TianHe and etc., the principles of APIT is triangle approach, assume that there are $\mathrm{n}$ anchor node which can communicate with the unknown node, the algorithm 
will traverse C_n^3 different triangles, and calculate the centroid of the overlap of the triangles as the evaluated location of the unknown node. The procedures of APIT are mainly about four steps as following:

- Anchor node information collecting phrase

Unknown nodes collecting the information about those anchor nodes which can communicate with it according to the information broadcasted by anchor nodes.

- PIT (Point in Test) executing phase

After the first phase, every unknown collected all the information of anchors nodes, so, the unknown node traverse every three of them and test if the unknown node in the triangle composed by the three anchor nodes.

- Grid scanning phase

If the unknown node in the triangle composed by some three anchor nodes, then the grids' count (initialized by 0 ) in the triangle will be crease by 1 .

- Centroid calculate phase

After the above three steps, we can find the entire grid who has the max count, then calculate the centroid of the polygon that composed by the grids.

\section{Innovation points of IMPROVED APIT algorithm}

Form the procedures and principles of APIT algorithm; we can see that there are three obvious disadvantages: (1) Average localization error rate cannot lower as communication radius increase; (2) The cover rate of APIT cannot reach 100\%; (3) Distribution-aware, the localization error rate and cover rate is different in different distributions. In order to solve this problem, this paper proposed an improved APIT algorithm called IMPROVED APIT algorithm, the main innovations of IMPROVED APIT are as following:

(1) Adjust the location of located nodes by formula 1 in order to reduce the localization error rate.

$$
\left\{\begin{array}{l}
\alpha=\left(x_{t}-x_{e}\right) /\left(x_{t}+x_{e}\right) \\
\beta=\left(y_{t}-y_{e}\right) /\left(y_{t}+y_{e}\right)
\end{array}\right.
$$

(2) Take the located nodes as the secondary anchor node in order to locate more nodes and thus the cover rate of the localization will be increased. And the secondary anchor node can relocate the nodes which cannot locate in the first circle.

(3) Give the special tackle of the node in inflexion points.

\section{Experimental result}

In order to test the performances of IMPROVED APIT localization algorithm, simulations has been done in this section, the simulation environment is MATLAB 2012, the premise of the simulations is shown as table 1 :

Table 1 Premises of simulations

\begin{tabular}{l|l|l}
\hline & Rectangle-shape & C-shape \\
\hline Experimental area & $1000 \times 1000 \mathrm{~m}^{2}$ & $1000,300,300,700$ \\
\hline Total number of nodes(n) & 200 & 200 \\
\hline Proportion of anchor nodes & 0.2 & 0.2 \\
\hline Communication radius(R) & $100,200, \ldots, 1000(\mathrm{~m})$ & $100,200, \ldots, 1000(\mathrm{~m})$ \\
\hline
\end{tabular}

This simulation test two kinds of distribution, the first kind of distribution is Rectangle-shape distribution, shown as figure 1 . And the second distribution is C-shape distribution, shown as figure 2. 


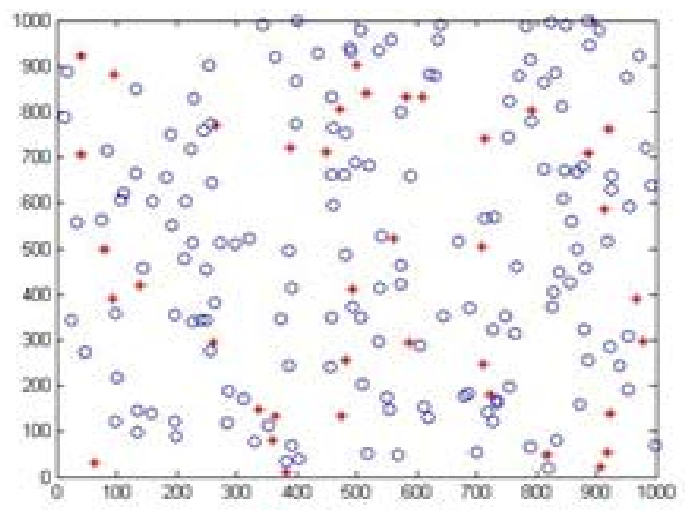

Fig 1 Nodes distribution in Rectangle-shape

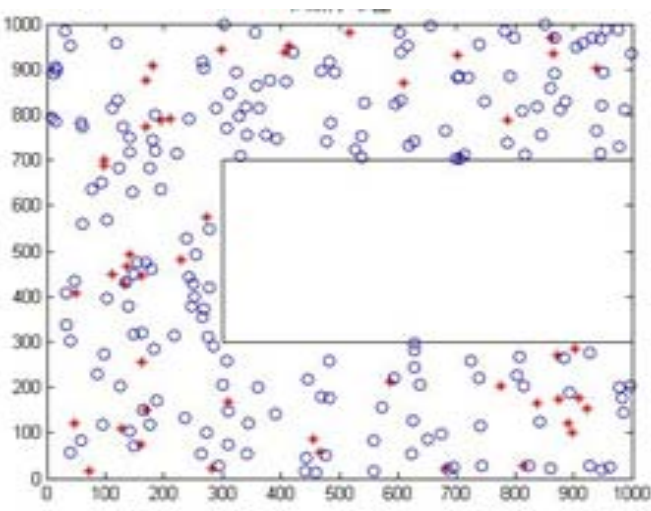

Fig 2 Nodes distribution in C-shape area area

Form figure 1 and figure 2, we can see that the distribution in MATLAB 2012 can represent the real environment in a certain degree. In the conditions of table 1, ten different experiments has been done, the experimental results are shown as figure 3 and figure 4 .

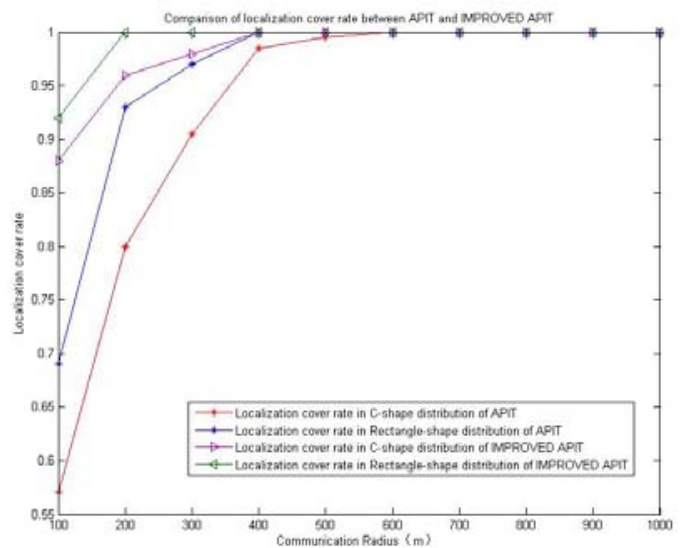

Fig 3 Localization Cover rate of APIT and IMPROVED APIT

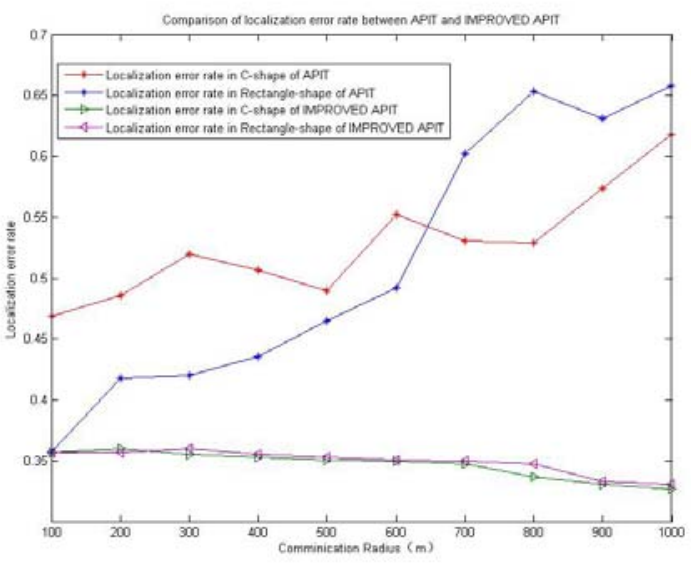

Fig 4 Localization Error rate of APIT and IMPROVED APIT

As shown in figure 3, the horizontal coordinate presents the communication radius and the vertical coordinate presents the localization cover rate, form figure 3, we can conclude that IMPROVED APIT's cover rate is higher that APIT's, and IMPROVED APIT's cover rate in C-shape is higher than Rectangle-shape, so the IMPROVED APIT is more suitable for core poisoning system. As shown in figure 4, we can conclude that the localization error rate of IMPROVED APIT is lower than APIT's, and the localization error rate of IMPROVED APIT is lower than the one in Rectangle-shape distributions, the conclusion shown that IMPROVED APIT is suitable for core poisoning systems.

How to use it in core monitoring system? How to set the parameters including communication radius and length of grid in core monitoring system, the important point analysis is shown as figure 5 and the results are shown as table 2. 


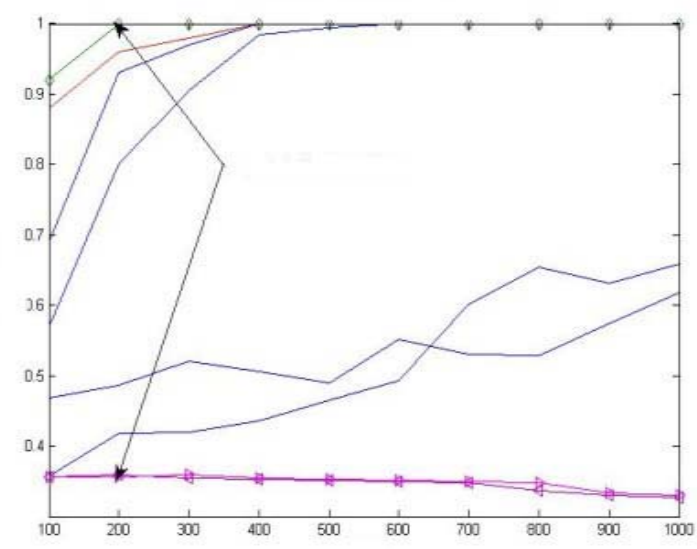

Fig 5 Parameter analysis of IMPROVED APIT algorithm

Table 2 Parameters of improved APIT in coal poisoning systemConclusion

\begin{tabular}{l|l}
\hline \multicolumn{1}{c|}{ Parameters } & \multicolumn{1}{c}{ Value } \\
\hline Length of the distribution area & L (unit: meter) \\
\hline Communication radius & L/5 (unit: meter) \\
\hline Length of grid & L/20 (unit: meter) \\
\hline \hline
\end{tabular}

This paper discussed the APIT algorithm and its application in core poisoning systems, according to the disadvantage of APIT in core poisoning systems, IMPROVED APIT algorithm is proposed in this paper, the most important contribution of IMPROVED APIT algorithm is its adaptive in C-shape distribution as the core well, the experimental research shows that IMPROVE APIT algorithm has a lower localization rate and a higher cover rate in core poisoning systems compared with APIT algorithm, the concreteness parameter in core poisoning system is also discussed in this paper, so, IMPROVED APIT algorithm has great significance when it is applied in core poisoning systems.

\section{Reference}

[1]. Amitangshu Pal. Localization Algorithms in Wireless Sensor Networks: Current Approaches and Future Challenges. Network Protocols and Algorithms. 45-74, 2010.

[2].J.Li, J. Jannotti, D. S. J. DeCouto, D. R. Karger and R. Morris. A Scalable Location Service for Geographic Ad-Hoc Routing. Proceedings of Sixth Annual International Conference on Mobile Computing and Networking. 120-130, 2000.

[3]. Yong Zhou, Xin Ao, Shixiong Xia. An Improved APIT Node Self-localization Algorithm inWSN. Proceedings of the $7^{\text {th }}$ World Congress on Intelligent Control and AutomationJune 25 27, 2008, Chongqing, China, 7582-7586.2012.

[4].Jizeng Wang. Improvement on APIT Localization Algorithms for Wireless Sensor Networks. 2009 International Conference on Networks Security, Wireless Communications and Trusted Computing.719-723, 2009.

[5].Hongbin Tan, Feng Liu. Research and Implementation of APIT Positioning Algorithm in WSN. $20129^{\text {th }}$ International Conference on Fuzzy Systems and Knowledge Discovery (FSKD 2012).2012. 